(2) Open Access Full Text Article

\title{
Spectrum of autoimmune vesiculobullous diseases in Iran: a I3-year retrospective study
}

\author{
Mohammadreza Sobhan \\ Mahmood Farshchian \\ Maryam Tamimi \\ Psoriasis Research Center, \\ Department of Dermatology, \\ Farshchian Hospital, Hamadan \\ University of Medical Sciences, \\ Hamadan, Iran
}

This article was published in the following Dove Press journal:

Clinical, Cosmetic and Investigational Dermatology

II January 2016

Number of times this article has been viewed

Background: Autoimmune bullous diseases (ABDs) represent a group of rare, acquired disorders characterized by overlapping features with involvement of the skin and mucous membranes, resistance to treatment, and potential lethality that comprise pemphigus, bullous pemphigoid (BP), epidermolysis bullosa, dermatitis herpetiformis, and linear immunoglobulin A bullous dermatosis.

Aim: The main aim of this study was to identify the epidemiologic characteristics and clinical courses of these common diseases in Hamadan, Iran. Few surveys have been carried out to demonstrate the whole spectrum of ABDs in the literature. Notably, in Hamadan we are the first.

Materials and methods: This 13-year retrospective study was designed to evaluate all of documented data obtained from hospitalized patients with ABDs at Farshchian Hospital from October 1999 to October 2012. We collected information on epidemiologic data, clinical aspects, histologic findings, and therapy prescribed. Data were analyzed using SPSS.

Results: Of 168 patients, $78 \%$ had pemphigus. The age of patients at presentation ranged from 1 month to 115 years, with a mean of $47.5 \pm 19.93$ years. Mucosal or skin involvement of ABDs was statistically significant $(P<0.001)$. The incidence of ABDs differed significantly based on anatomic location $(P=0.003)$. We documented three deaths.

Conclusion: Compared to previous literature, our findings showed equal epidemiologic properties in Iran. Although pemphigus was the most common ABD followed by BP, it is expected that in line with the global trend, an increase in BP will be driven by population aging in Iran.

Keywords: autoimmune bullous disease, pemphigus, prevalence

\section{Introduction}

Autoimmune bullous diseases (ABDs) represent a group of rare, acquired disorders characterized by overlapping features, resistance to treatment, and potential lethality. ${ }^{1}$ The most important pathophysiologic mechanisms in the skin of these patients have been identified as disruption of the host immune system, which leads to clinical blister formation. This group of diseases includes bullous pemphigoid (BP), dermatitis herpetiformis, linear immunoglobulin A (IgA) bullous dermatosis (LABD), and epidermolysis bullosa (EB). ${ }^{1,2}$

Many published epidemiologic studies have focused on pemphigus, but few have encompassed the total spectrum of ABDs. Few surveys have been carried out to demonstrate the entire spectrum of ABDs in Iran. Notably, in Hamadan, Iran, we are the first. The main aim of this study was to identify the epidemiologic characteristics and clinical courses of these common diseases in Hamadan. 


\section{Materials and methods}

The descriptive, retrospective, cross-sectional study was carried out in the Dermatology Department of Farshchian Hospital, Hamadan University of Medical Sciences, the regional secondary referral center for cutaneous diseases. This study conformed to the ethical guidelines of the 1975 Declaration of Helsinki. Hamadan University of Medical Sciences Institutional Review Board approved the research. Patient consent is not required for this retrospective study. All patients hospitalized at the department between October 1999 and October 2012 with a clinical diagnosis of ABDs were examined to characterize epidemiologic aspects of ABDs in Hamadan. Data were collected by means of record review, and the parameters of interest were surveyed in a protocol developed by the investigators. The criteria used for making the diagnosis were clinical features along with histopathologic findings; in addition, Direct immunofluorescence was properly used in many patients (but not all cases) when necessary. Documented information of bedridden patients included age, sex, bedridden duration, BD subtype, treatment modality, treatment duration, family history, mortality rate, and histopathologic results. A number of lesions approved on different sectors of the body and on the mucous membranes were detected. Details of documents were reviewed with regard to the clinical course of each patient at the onset of the disease, including age of patient in which the disease began, primary affected site, and season at onset. Thereby, the duration of the disease was calculated, ie, the interval between the onset of disease until the present. In this period, first complete remission and recurrence rate were noticed. Patients with incomplete or missing data in their records were excluded. The number of files excluded due to incompleteness was trivial, because we tried our best to avoid missing data. For this reason, two of us separately kept a careful check on each file for a second time to ensure that the findings were accurate. Therefore, we made sure we did not miss any important details.

Data were analyzed using SPSS version 20.0 (IBM Corporation, Armonk, NY, USA). Frequencies and percentages were calculated for qualitative variables. Means, mode, and standard deviation were calculated for quantitative variables. Student's $t$-test and the $\chi^{2}$ test were used for statistical analysis. Fisher's exact test was performed as necessary. Confidence rate higher than $95 \%$ was considered significant $(P<0.05)$.

\section{Results}

In sum, 168 patients were diagnosed to have ABDs. The characteristics of the patients according to the type of disease are given in Table 1. There were 78 male patients (46.4\%) and 90
Table I Characteristics of the patients with different autoimmune bullous diseases (ABDs)

\begin{tabular}{lllll}
\hline ABD & $\begin{array}{l}\text { Cases, } \\
\mathbf{n}(\%)\end{array}$ & $\begin{array}{l}\text { Male:female } \\
\text { ratio }\end{array}$ & $\begin{array}{l}\text { Mean age at } \\
\text { onset } \\
\text { (years } \pm \text { SD) }\end{array}$ & $\begin{array}{l}\text { Mean age at } \\
\text { presentation } \\
\text { (years } \pm \text { SD) }\end{array}$ \\
\hline Pemphigus & I3I (78) & I:I.II & $43.30 \pm 17.20$ & $45.44 \pm 17.30$ \\
BP & $2 I(12.5)$ & I:I.I & $71.19 \pm 14.06$ & $72.23 \pm 13.77$ \\
DH & II (6.5) & I:4.5 & $38.18 \pm 18.90$ & $40.27 \pm 18.42$ \\
EB & $5(3)$ & $1: 4$ & $5.2 \pm 10.54$ & $13.42 \pm 10.44$ \\
LABD & 0 & - & - & - \\
Total & I68 (I00) & I:I.I5 & $45.32 \pm 20.42$ & $47.50 \pm 19.93$ \\
\hline
\end{tabular}

Abbreviations: SD, standard deviation; $\mathrm{BP}$, bullous pemphigoid; $\mathrm{DH}$, dermatitis herpetiformis; $\mathrm{EB}$, epidermolysis bullosa; $\mathrm{LABD}$, linear IgA bullous dermatosis.

female patients (53.6\%), with a male:female ratio of 1:1.15. We found that women had a slightly higher rate of ABDs than men, which was not statistically significant $(P=0.119)$. The distribution of patients in age-groups is shown in Figure 1. The youngest patient was a 1-month-old newborn with EB at birth, and the oldest patient was 115-years-old and had pemphigus. The most frequent ages for ABDs were 24 and 61 years, each of which represented seven patients $(4.2 \%)$. The majority of the patients $(20.8 \%)$ were in the fourth decade of their life.

Characteristics of the diseases are shown in Table 2. EB represented the most long-standing symptoms. The sites of involvement in the first episode were as follows: the two main sites were oral mucosa, with 69 cases, and front of the trunk, with 47 cases. Lower frequencies were seen on the head, posterior trunk, lower extremities, upper extremities, neck, and genitals with 29, 28, 19, 15, three, and one, respectively. The sum of these frequencies was greater than 168 , which reflected disseminated lesions in some patients in the first episode. The incidence of ABDs differed significantly based on anatomic location $(P=0.003)$. Phenotypes of mucocutaneous involvement in ABD patients revealed that 86 cases showed involvement of mucous membranes and skin surface concomitantly. In 59 patients, skin lesions were seen alone, and in 23 cases mucosal lesions were seen alone, which was statistically significant $(P<0.001)$.

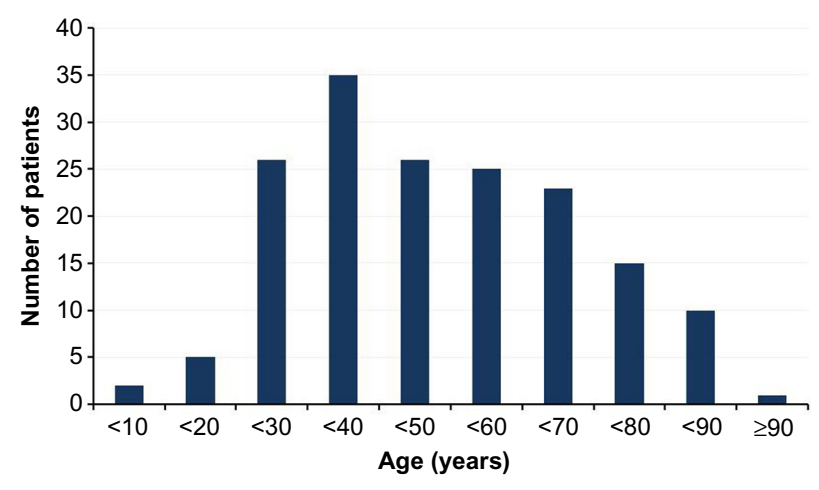

Figure I Distribution of patients in age-groups. 


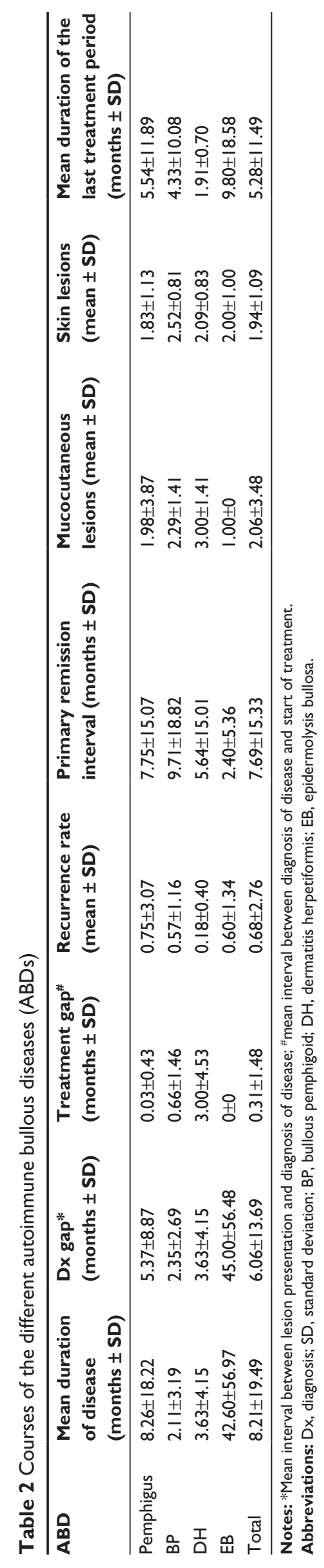

The shortest interval between the presentation of lesions and diagnosis of disease was 1 month, whereas the longest was 121 months. That means this patient had been treated based on diagnoses other than $\mathrm{ABDs}$ for more than 10 years.

The first manifestations occurred most often in summer and autumn (60\%), followed by spring and winter. The onset of disease and season did not correlate statically $(P<0.273)$. Positive family history was seen only in three pemphigus patients. Other cases in our study had not any history of ABDs in their family, which was not statistically significant $(P=0.979)$. A mismatch between clinical and histopathologic diagnosis was seen only in one pemphigus case.

With a mean of 9.8 months, EB had the most prolonged treatment duration. The most prevalent modality for treatment of ABDs was pulse therapy; 103 patients (61.3\%) received corticosteroids as pulse therapy and 62 patients (36.9\%) were treated with oral corticosteroids. Cyclophosphamide was added to steroids in 93 cases (55.4\%) and azathioprine in nine cases $(5.4 \%)$ as adjuvant therapy. The remaining 66 patients $(39.2 \%)$ were treated with corticosteroids alone. Only three patients, including BP, dermatitis herpetiformis, and EB, were hospitalized without any treatment, and were just admitted for confirming the diagnoses.

All of the three cases who died from ABDs in our study had pemphigus: a 52-year-old woman and two men aged 45 and 75 years. The woman was treated with corticosteroid as pulse therapy in conjunction with cyclophosphamide. The men were treated with high-dose oral corticosteroids without adjuvant drugs. All of them died in our hospital from septicemia due to treatment complications.

\section{Discussion}

ABDs are uncommon, and the exact incidence and prevalence depend on the population studied. Similar to an earlier observation from our country, ${ }^{3}$ we found a female predilection in each group of ABDs. This was in common with other countries like Tunisia ${ }^{4}$ and Kuwait. ${ }^{5}$ The mean age of our patients at onset of the disease was $45.32 \pm 20.42$ years, which was in line with a previous Iranian result ( $44.99 \pm 17.35$ years). ${ }^{3}$ The mean age of the patients at presentation to our hospital (47.5 years) was comparable to Al-Sabah Hospital in Kuwait, with a similar ethnic background (42 years). ${ }^{5}$ It is noticeable that the differences in threshold for hospitalization of $\mathrm{ABD}$ patients in different countries may explain some of the variation in results. Indeed, ethnic, environmental, or genetic variation emphasized the different results in various countries.

What really allowed unambiguous differentiation of the ABDs was the characterization of their histology. ${ }^{6}$ Our 
results confirmed that our clinical diagnosis, almost always, was consistent with histopathologic findings. This evidence would provide a major clue as to the importance of clinical experience of specialists. We found that the average primary remission interval in all groups of $\mathrm{ABDs}$ was lower than 1 year. Therefore, repeated physical examinations during regular visits in the first year after treatment may improve the quality of management.

\section{Pemphigus}

We reported pemphigus as the most common $\mathrm{ABD}$ in Hamadan. It has been proposed by other investigators in some other countries, including northwestern Romania (58.6\%), ${ }^{7}$ Tunisia (52.9\%), ${ }^{4}$ Kuwait $(47 \%),{ }^{5}$ and Thailand $(63.3 \%) .{ }^{8}$ Also, two Iranian studies reported the percentage as $86.1 \%{ }^{3}$ and $83.67 \% .{ }^{9}$ The mean age of pemphigus patients at the onset of disease has been reported as $38,{ }^{10} 42,{ }^{11} 45.8,{ }^{9}$ and $53^{7}$ years, and was 43.3 years in our study. The mean age of our pemphigus patients at presentation (45.44 years) was consistent with earlier research, at 44.6, ${ }^{12} 45.6,{ }^{13} 46,{ }^{14,15} 52.5,{ }^{16}$ and $62.43^{17}$ years. Although it was not statistically significant, we found a female predominance in the pemphigus patients (male:female $=1: 1.11$ ). There have been similar results in other provinces of Iran and other countries. By a review of the literature, the male:female ratio in pemphigus was $1.1: 1,{ }^{9} 1: 1.11,{ }^{17} 1: 1.33,{ }^{10,16}$ $1: 1.5,{ }^{11,15} 1: 1.53,{ }^{12} 1: 1.64,{ }^{18} 1: 1.75,{ }^{7} 1: 1.8,{ }^{19}$ and $1: 2.23 .{ }^{14}$

In our pemphigus cases, the oral mucosa was the most common primary site of involvement in the beginning of disease (42.7\%). This was consistent with Farshchian and Pilehvar (34.3\%), ${ }^{18}$ Salmanpour et al (59.3\%), ${ }^{10}$ Chams-Davatchi et al $(62 \%),{ }^{11}$ and Sadr-Ashkevari et al $(63 \%) .{ }^{13}$ In the course of the disease, pemphigus most often affected (60\%) both the mucous membranes and the skin in our study. This was similar to some other studies: $64 \%,{ }^{17} 67.8 \%,{ }^{19}$ and $70 \% .{ }^{11}$ However, Abdolsamadi et al found that the oral mucosa in $44.9 \%$ of pemphigus patients was exclusively affected at presentation time, and oral and skin lesions were seen simultaneously in only $16.7 \%$ of cases. ${ }^{12}$ Farshchian and Pilehvar detected oral lesions with or without skin lesions in $83.8 \%$ of pemphigus patients. ${ }^{18}$ The mean duration of pemphigus (from the beginning of lesions until the last presentation) was 8.26 months in our findings. In contrast, Chams-Davatchi et al found 4.5 years for duration of the disease, and their mean follow-up duration was 3.8 years. ${ }^{11}$ Also, Tsankov et al showed that the mean duration of the pemphigus was 8.64 years for women and 6.42 years for men. ${ }^{17}$

Pemphigus is a rare but chronic disease, and is associated with significant morbidity and mortality. In the present study, there were three deaths $(2.29 \%)$ from a total of 131 pemphigus cases in the period of 13 years. This was in the range of other surveys, which demonstrated 1\%-20\%: $2.3 \%$ (36 deaths in 1,560 patients), ${ }^{12} 7.14 \%$ (eight deaths in 112 patients), ${ }^{15} 7.65 \%$ (18 deaths in 235 patients), ${ }^{20}$ $9.45 \%$ (seven deaths in 74 patients), ${ }^{17} 10.8 \%$ (four deaths in 37 patients), ${ }^{18}$ and $20 \%$ (eleven deaths in 55 patients). These eleven deaths were observed in Kerman, where Shamsadini et al showed that mortality rates were $7.3 \%$, $13.2 \%$, and $38.5 \%$ at 2,6 , and 10 years, respectively. ${ }^{14}$ Two other prospective studies, from Tehran $(6.2 \%)^{11}$ and Taiwan $(10.31 \%),{ }^{16}$ have validated the high mortality rate due to pemphigus. Huang et al found that overall mortality among pemphigus patients was two times (2.36-fold) greater than that of the general population. Therefore, the survival rate in patients diagnosed with pemphigus was significantly lower than that expected in the general population. ${ }^{16}$ We found that septicemia was the commonest cause of deaths in pemphigus patients (100\%), consistent with earlier studies. ${ }^{11,12,15,16,18,20}$ The main information about the prognosis of pemphigus was from Halaji et al. They showed that old age, male sex, interval between beginning of lesions and diagnosis of more than 6 months, and more than ten skin lesions were accompanied by poor prognosis. ${ }^{21}$

\section{Bullous pemphigoid}

Although BP was the second-most common $\mathrm{ABD}$ and the most common subepidermal BD in our series, predictable BP prevalence should be much higher than the number that was referred to us. It is important to remember the nonspecific BP clinical symptoms that may lead to underestimation. These points highlight the value of clinical diagnosis of BP in older patients. The mean age of BP patients was 82.6 years, ${ }^{22} 80,{ }^{23}$ $79.6,{ }^{24}$ and $74.5^{25}$ years in France, the UK, Portugal, and the USA, respectively. These were a little higher than our result (72 years), though in Thailand it was 69.3 years. ${ }^{8}$ Likewise, another study in Iran reported 64.1 years. ${ }^{9}$ This would be interpreted as due to higher life expectancy in developed countries. This implies that as life expectancy increases, the incidence and the duration of disease may increase. In this way, a study has pointed out that life expectancy for Iran or Thailand is much lower than developed countries. ${ }^{26}$ Furthermore, we found that incidence of BP increased significantly with age. Several surveys confirmed this finding. ${ }^{8,22,24,27-29}$ Most authors agreed on general opinion considering increased BP incidence is attributable to a greater proportion of older persons in the general population. ${ }^{25}$ Broader analytic studies have been carried out, one in the USA and a population-based 
cohort study in the UK. Both confirmed that incidence of BP increased over time across age-groups. ${ }^{23,25}$

A review of the literature revealed that $\mathrm{BP}$ was the most frequent ABD in the USA, ${ }^{25} \mathrm{Canada}^{30}$ and Europe: France, ${ }^{22}$ the UK, ${ }^{23}$ and Germany. ${ }^{27}$ Wong and Chua found that BP in Singapore was diagnosed three times more frequently than pemphigus. ${ }^{31}$ This is in contrast with other regions in which authors identified BP as the second-most prevalent ABD. For example, the ratio of BP to total ABDs was reported as $11.6 \%$ in Tehran, ${ }^{3} 22 \%$ in Kuwait, ${ }^{5} 23.6 \%$ in Tunisia, ${ }^{4} 34.5 \%$ in Romania, ${ }^{7}$ and $12.5 \%$ in our series. Therefore, BP is much more common in developed countries rather than developing countries like Iran. Bertam et al diagnosed 27 BP cases (65\%) among a total of $41 \mathrm{ABD}$ patients in Germany. The mean age at disease onset was 74 years in this European city. ${ }^{27}$ North East Scotland appeared to have a relatively high incidence rate for Europe. The mortality rate due to BP in this region was considerable: $48 \%$ of patients with BP died within 2 years of diagnosis. ${ }^{28}$ Joly et al found that the incidence of BP in France had increased threefold in the last 15 years. Relative to expected age- and sex-specific overall death rates in the general population in France, there was a more than sixfold excess of mortality among BP patients. That was assessed from a prospective cohort study. ${ }^{22}$ Among bullous skin diseases, pemphigoid was the leading cause of mortality in Canada. ${ }^{30}$ Although, BP mortality was much lower in the USA than Canada and Europe, it was higher than previous estimates. ${ }^{25}$

Although ultrapotent topical corticosteroids are the firstline therapy for $\mathrm{BP}^{32}$ all of our admitted patients, even $\mathrm{BP}$ cases, received systemic corticosteroids, and most of the protocols involved pulse therapy. The cause of this paradox is reasonable in a way that is clear. Mild-to-moderate BP patients usually do not need to be admitted. Therefore, our patients needed more aggressive treatment modalities. Jowkar et al similarly showed that systemic drugs were administered to all BP patients; moreover, prednisolone monotherapy was the most frequent medication in a Shiraz hospital. ${ }^{9}$

\section{Epidermolysis bullosa}

We detected $\mathrm{EB}$ as a very rare $\mathrm{ABD}(3 \%)$. Other authors have confirmed it as $0.5 \%,{ }^{3} 0.6 \%,{ }^{4}$ and $2.3 \% .{ }^{5}$ EB cases were the youngest patients at the onset of the disease, but it was taking more time for diagnosis compared with other ABDs. This would be explained by delayed presentation to physicians or misdiagnosis with other infantile or childhood vesiculobullous diseases. Surprisingly, this time-consuming diagnosis was followed by immediate treatment. Noticeably, we found that EB had the longest disease course.

\section{Linear IgA bullous dermatosis}

LABD is rare in Western Europe, affecting mainly adults, but is more prevalent in Tunisia ${ }^{4}$ and Kuwait, ${ }^{5}$ with more children involved. Actually, it is the commonest ABD of childhood in Tunisia. ${ }^{3}$ Daneshpazhooh et al found that in Iran, LABD was seen in only $0.4 \%$ of patients. ${ }^{3}$ Also, it is rare in Singapore. ${ }^{31}$ We observed no case of hospitalized LABD in our study.

\section{Limitations}

Data obtained by reviewing inpatient and outpatient records revealed that some cases may not reach our department, because they may be treated without hospitalization. Prospective studies or outpatient-plus-inpatient sampling may partially correct this bias.

\section{Conclusion}

Our findings compared to previous literature showed equal epidemiologic properties in Iran. Skin or mucosal involvement and anatomic location of lesions were two identifiable variables in the ABDs, with statistically significant value. Systemic corticosteroids, either with or without adjuvant drugs, were ordered for all of the patients, and most of them were cured. In our hospital, three patients died due to septicemia. In general, in most regions of our country, the whole spectrum of ABDs has not been described completely. Indeed, the epidemiologic characteristics must be distinguished in each state of Iran to demonstrate the therapeutic approach.

\section{Disclosure}

The authors report no conflicts of interest in this work.

\section{References}

1. Yancey KB, Fairley JA. Introduction to the milestones in autoimmune bullous diseases. J Invest Dermatol. 2008;128:E15.

2. Liu Z, Rubenstein DS. Pathophysiology of autoimmune bullous diseases. J Invest Dermatol. 2008;128:E22-E24.

3. Daneshpazhooh M, Chams-Davatchi C, Payandemehr P, Nassiri S, Valikhani M, Safai-Naraghi Z. Spectrum of autoimmune bullous disease in Iran: a 10-year review. Int J Dermatol. 2012;51:35-41.

4. Zaraa I, Kerkeni N, Ishak F, et al. Spectrum of autoimmune blistering dermatoses in Tunisia: an 11-year study and a review of the literature. Int J Dermatol. 2011;50:939-944.

5. Nanda A, Dvorak R, Al-Saeed K, Al-Sabah H, Alsaleh QA. Spectrum of autoimmune bullous diseases in Kuwait. Int J Dermatol. 2004;43: 876-881.

6. Stanley JR, Amagai M. Autoimmune bullous diseases: historical perspectives. J Invest Dermatol. 2008;128:E16-E18.

7. Baican A, Baican C, Chiriac G, et al. Pemphigus vulgaris is the most common autoimmune bullous disease in northwestern Romania. Int $J$ Dermatol. 2010;49:768-774.

8. Kulthanan K, Chularojanamontri L, Tuchinda P, Sirikudta W, Pinkaew S. Prevalence and clinical features of Thai patients with bullous pemphigoid. Asian Pac J Allergy Immunol. 2011;29:66-72. 
9. Jowkar F, Sadaty MS, Tavana S, Agah MA. Epidemiology of autoimmune bullous diseases and therapeutic modalities during a 10 year period in Iran. Acta Dermatovenerol Croat. 2014;22:246-249.

10. Salmanpour R, Shahkar H, Namazi MR, Rahman-Shenas MR. Epidemiology of pemphigus in south-western Iran: a 10-year retrospective study (1991-2000). Int J Dermatol. 2006;45:103-105.

11. Chams-Davatchi C, Valikhani M, Daneshpazhooh M, et al. Pemphigus: analysis of 1209 cases. Int J Dermatol. 2005;44:470-476.

12. Abdolsamadi HR, Abdollahzade S, Bakianian-Vaziri P, Beheshti A, Shafigh E, Vahedi M. Epidemiology of pemphigus in Tehran, Iran: a 20-year retrospective study. J Dent Res Dent Clin Dent Prospects. 2007;1:108-113.

13. Sadr-Ashkevari S, Shams-Guilani J, Dorjani A, et al. Pemphigus in Guilan: clinical and epidemiologic features of 126 hospitalized patients. Iran J Dermatol. 2005;8:104-109.

14. Shamsadini S, Fekri AR, Esfandiarpoor I, et al. Determination of survival and hazard functions for pemphigus patients in Kerman, a southern province of Iran. Int J Dermatol. 2006;45:668-671.

15. Aboobakr J, Morar N, Ramdial PK, Hammond MG. Pemphigus in South Africa. Int J Dermatol. 2001;40:115-119.

16. Huang YH, Kuo CF, Chen YH, Yang YW. Incidence, mortality, and cause of death of patients with pemphigus in Taiwan: a nationwide population-based study. J Invest Dermatol. 2012;132:92-97.

17. Tsankov N, Vassileva S, Kamarashev J, Kazandjieva J, Kuzeva V. Epidemiology of pemphigus in Sofia, Bulgaria. A 16-year retrospective study. Int J Dermatol. 2000;39:104-108.

18. Farshchian M, Pilehvar M. Evaluation of characteristics of pemphigus patients hospitalized in the dermatology ward of Sina Hospital Hamadan Iran during 1992-1995. Sci J Hamadan Univ Med Sci Health Serv. 2001;8:24-28.

19. Esmaili N, Chams-Davatchi C, Valikhani M, et al. Assessment of the therapeutic benefit of oral prednisolone and common adjuvant therapy in stage II of randomized controlled trial study for management of pemphigus vulgaris. Arch Iran Med. 2014;17:626-628.

20. Nair SP. A retrospective study of mortality of pemphigus patients in a tertiary care hospital. Indian J Dermatol Venerol Leprol. 2013;79: 706-709.
21. Halaji Z, Esmaeili N, Moeineddin F, Zeinali F, Alami L, ChamsDavatchi C. Prognostic factors of pemphigus vulgaris disease: a study on 119 patients. Tehran Univ Med J. 2009;66:913-918.

22. Joly P, Baricault S, Sparsa A, et al. Incidence and mortality of bullous pemphigoid in France. J Invest Dermatol. 2012;132:1998-2004.

23. Langan SM, Smeeth L, Hubbard R, Fleming KM, Smith CJ, West J. Bullous pemphigoid and pemphigus vulgaris - incidence and mortality in the UK: population based cohort study. BMJ. 2008;337:a180.

24. Teixeira VB, Cabral R, Brites MM, Vieira R, Figueiredo A. Bullous pemphigoid and comorbidity: a case-control study in Portuguese patients. An Bras Dermatol. 2014;89:274-278.

25. Brick KE, Weaver CH, Lohse CM, et al. Incidence of bullous pemphigoid and mortality of patients with bullous pemphigoid in Olmsted County, Minnesota, 1960 through 2009. J Am Acad Dermatol. 2014;71: 92-99.

26. Wang H, Dwyer-Lindgren L, Lofgren KT, et al. Age-specific and sexspecific mortality in 187 countries, 1970-2010: a systematic analysis for the Global Burden of Disease Study 2010. Lancet. 2012;380: 2071-2094.

27. Bertram F, Bröcker EB, Zillikens D, Schmidt E. Prospective analysis of the incidence of autoimmune bullous disorders in Lower Franconia, Germany. J Dtsch Dermatol Ges. 2009;7:434-440.

28. Gudi VS, White MI, Cruickshank N, et al. Annual incidence and mortality of bullous pemphigoid in the Grampian region of North-East Scotland. Br J Dermatol. 2005;153:424- 427.

29. Marazza G, Pham HC, Schärer L, et al. Incidence of bullous pemphigoid and pemphigus in Switzerland: a 2 year prospective study. $\mathrm{Br} J$ Dermatol. 2009;161:861-868.

30. Baibergenova AT, Weinstock MA, Shear NH. Mortality from acquired bullous diseases of skin in Canadian adults 2007-2009. Int J Dermatol. 2012;51:1325-1328.

31. Wong SN, Chua SH. Spectrum of subepidermal immunobullous disorders seen at the National Skin Centre, Singapore: a 2-year review. Br J Dermatol. 2002;147:476-480.

32. Bernard P, Charneux J. Bullous pemphigoid: a review. Ann Dermatol Venerol. 2011;138:173-181.
Clinical, Cosmetic and Investigational Dermatology

\section{Publish your work in this journal}

Clinical, Cosmetic and Investigational Dermatology is an international, peer-reviewed, open access, online journal that focuses on the latest clinical and experimental research in all aspects of skin disease and cosmetic interventions. All areas of dermatology will be covered; contributions will be welcomed from all clinicians and

\section{Dovepress}

basic science researchers globally. This journal is indexed on CAS The manuscript management system is completely online and includes a very quick and fair peer-review system, which is all easy to use. Visit http://www.dovepress.com/testimonials.php to read real quotes from published authors. 